\title{
Hardware and software for tachistoscopy: How to make accurate measurements on any PC utilizing the Microsoft Windows operating system
}

\author{
COLLIN J. MCKINNEY, EARL R. MACCORMAC, and KATHLEEN A.WELSH-BOHMER \\ Duke University Medical Center, Durham, North Carolina
}

\begin{abstract}
Methods for automated stimulus display and accurate response time measurement with IBMcompatible PCs are of great importance in cognitive research designs. Accurate measurements of reaction times are required to interpolate other measures, such as speed of mental processing. We present a description of hardware and software that displays stimulus images and performs reaction timing that is not dependent on PC performance characteristics. This is accomplished by electronically bypassing timing errors normally inherent to the operation of the computer. A high-precision external timer measures the time between stimulus onset and a subject's push-button response while a video blanking circuit controls the video presented to the monitor screen. Two options for accurately detecting stimulus onset are presented: (1) A photodetector can be used to sense the actual onset of the stimulus on a secondary video monitor screen; (2) the video blanking circuit can provide a signal coincident with the initiation of video to the monitor. Both methods result in a system timing accuracy of $100 \mu \mathrm{sec}$.
\end{abstract}

Psychological research often requires the accurate measurement of reaction times in response to visual stimuli. In many cases, reaction time measurements are used to give an indication of subject involvement in the task, to evaluate the performance of the subject, and to help discriminate among various brain functions. Currently, the visual stimuli for projects performed in our laboratory include words, simple geometric figures, and complex fractal images. Future projects will most certainly involve such images as digitally rendered photographs or other more complex images in three dimensions or in motion. Computer-based methods for displaying images and measuring response times are desirable, because of the degree of automation that is available and because of the ease of modifying the stimulus through simple programming. IBM PC-compatible computers have found widespread use and provide a reasonable platform for laboratory measurements.

However, computer-based methods for providing stimuli and for measuring response times have various limitations, many of which have been described in the literature (Dutta, 1995; Gabrielsson \& Jarvella, 1990; Mogg \& Bradley, 1995; Palya, Walter, \& Chu, 1995; Paredes, Miller, \& Creeger, 1990; Rodgers, Schneider, Pitcher, \& Zuccolotto,1995; Segalowitz \& Graves, 1990). The major

This research was supported in part by grants from the National Institute of Aging (AG05128, AG09997). The authors gratefully acknowledge Christen M. Cullen for her work in preparing lists of stimuli and for assisting in the testing of the initial hardware and software and Dr. David Madden for his insightful input. Correspondence concerning this article should be addressed to C. J. McKinney, Box 3808, Department of Radiology, Duke University Medical Center, Durham, NC 27710 (e-mail: collin@petsparc.mc.duke.edu) limitation that arises from the use of a PC is that the accuracy of time measurements is highly dependent on the PC's type, speed, external hardware, and software. For example, the commercially available Micro Experimental Laboratory (MEL) system has been shown to have inaccuracies of between \pm 1.5 and $\pm 6.5 \mathrm{msec}$, depending on the hardware used (Mogg \& Bradley, 1995). Various inaccuracies can result when utilizing a PC's internal timers, because of a lack of complete control over the microprocessor's activities. A method using assembly language control over the serial port resulted in a reported resolution of $1 \mathrm{msec}$ (Gabrielsson \& Jarvella, 1990), but the ability to maintain this accuracy while the processor is performing other tasks was not fully addressed, and the developers acknowledged the highly machine-dependent nature of the software program. Graves and Bradley (1991) have indicated that reliable millisecond timing can be performed on most PCs utilizing assembly language codes under DOS (Graves \& Bradley, 1991).

Our particular projects require the use of complex image stimuli and a simple user interface, both of which are easily supported within Microsoft Windows, as opposed to the DOS environment. Therefore, a flexible method for presenting stimuli and for accurately measuring reaction times, using an IBM-compatible PC running Microsoft Windows, was desired for use in cognitive function studies. Several of the main design goals of this project include (1) the ability to avoid timing inaccuracies from differing equipment hardware specifications (i.e., processor speeds, operating system overhead, video processing speeds, video scan rates, etc.), so that 100 $\mu$ sec timing resolution can be obtained under all conditions; (2) the ability to make measurements from software running under the Windows operating system; (3) the use 
of hardware that is external to the computer, so that it can be transferred between computers without the use of tools and so that it can be used with laptop as well as with desktop computers; (4) the use of a common, easy-to-use programming language such as BASIC, so that it can be easily understood by other users and so that it can be modified readily; and (5) the ability to use complex visual stimuli that are either transferred by the cut-and-paste method from other computer application software packages or digitally rendered by scanning images, facilitated by the use of Windows.

\section{SYSTEM DESIGN}

\section{Hardware}

Event timing on a PC can be troublesome, especially when a multitasking operating system such as Windows is utilized. In this environment, the processor is continually being interrupted in order to perform other tasks, even when many of the extraneous features have been disabled, making accurate timing difficult, if not impossible. Therefore, it was decided that the use of an external time base and counter would provide the most accurate and reliable results. The time measurement accuracy of this approach is a function of the stability of the crystalcontrolled time-base oscillator and the digital resolution of the counter (number of bits). Commercially available time-base oscillators generally have stabilities that are several orders of magnitude smaller than the minimum resolution required for tachistoscopic measurements.

Many counter/timer boards that plug into a PC expansion slot are available, but in order to make the system easily transportable between computers, a unit that con- nects to the standard parallel printer port found on all computers was chosen (ComputerBoards, Inc., model PPIO-CTR06). This particular board has an integral 10$\mathrm{MHz}$ time base, an Intel 8254 with three 16-bit down counters, and 24 TTL I/O ports. The stability of the timebase oscillator used in this particular board is specified by the manufacturer to be $100 \mathrm{ppm}$ with $5 \mathrm{psec}$ maximum jitter. The three counters are connected in series and are used to divide the time-base signal by software presettable constants. Hardware was designed to control the gating of the counters through the use of external start and stop signals, including a remotely located stop pushbutton (see Figure 1). This circuitry includes debouncing for the pushbutton switch and a flip-flop that latches the gate control output high (after a start pulse is received) until a stop is received from the pushbutton or a clear is received from the computer. An LED is used to indicate pushbutton activity.

Originally, the computer provided the start signal (through one of the TTL I/O ports) just prior to writing the stimulus on the screen. A significant variable delay time exists, however, between this start signal and the time that the stimulus is actually written to the screen. This delay time, dependent on the computer hardware and software, causes a large amount of variability in measured reaction times. Use of a 75-MHz Pentium-based PC running Windows 3.1 with control software written in VisualBasic and displaying six 72-point Times Roman characters in the center of the screen resulted in the distribution of delay times shown in Figure 2. These data were measured with a phototransistor, to measure the time interval between the start pulse from the computer and the appearance of the first character on the screen (Mogg \&

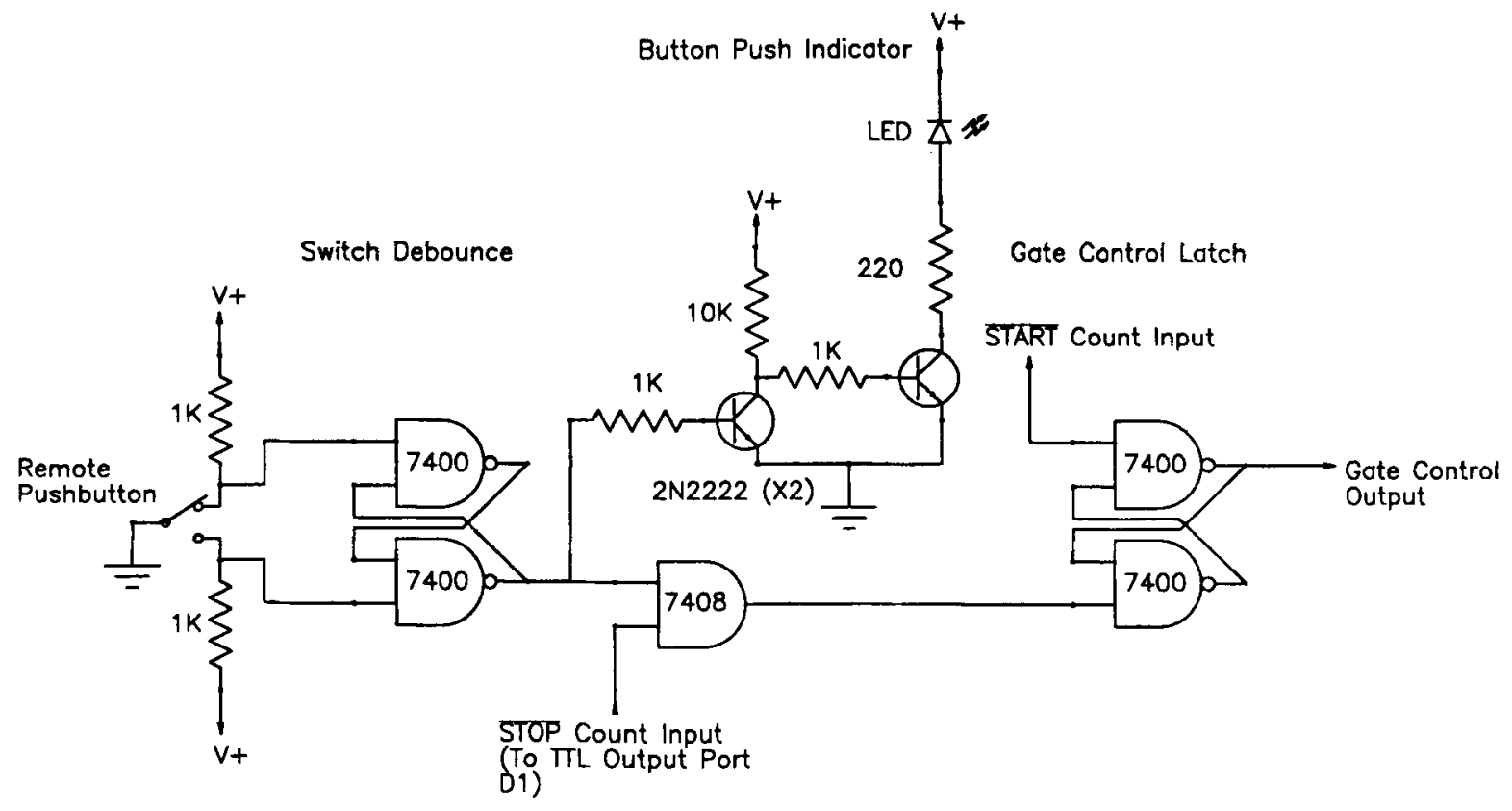

Figure 1. Remote pushbutton circuitry. 


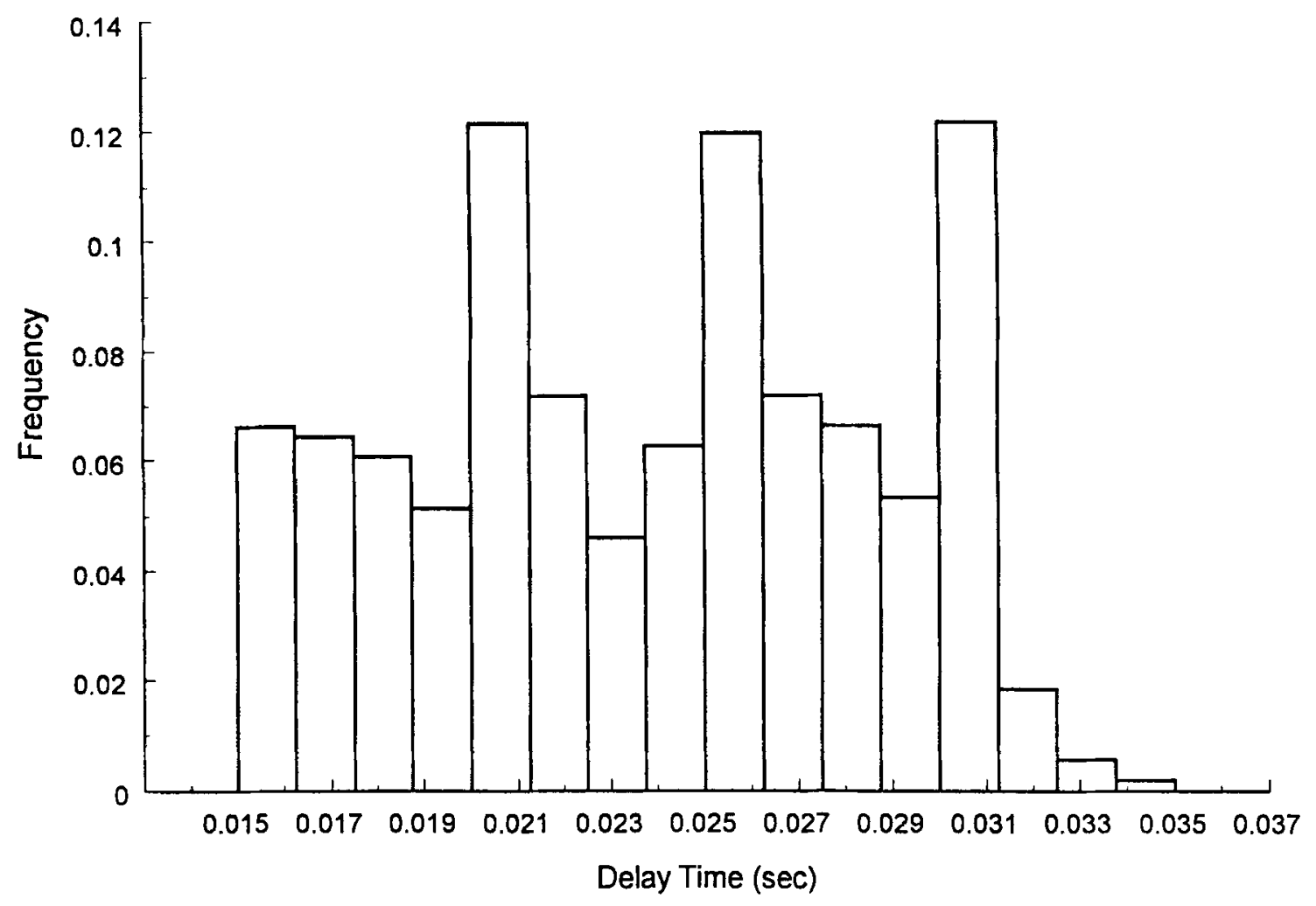

Figure 2. Delay time distribution.

Bradley, 1995). This delay time is generally a function of (1) processor speed and bus width, (2) video card parameters (i.e., memory, accelerator speed, etc.), (3) processor overhead and concurrent processing of interrupts, and (4) timing of image initiation with respect to raster position.

In order to alleviate these problems and to allow the hardware and software to run on a wide variety of IBMcompatible PCs, the circuitry shown in Figure 3 was developed. This circuitry replaces the start signal from the computer with a start signal based on the actual appearance of the image on the monitor screen. Our test configuration requires two video display monitors. One displays the stimulus to the subject, whereas the other serves as the experiment control monitor. A video buffer/splitter (Raritan Computer, Inc., model DRV1X2) is used to drive both monitors from a single computer. A photodetector, placed on the control monitor, detects the appearance of the stimulus image. Specifically, the photodetector consists of a pair of phototransistors in a differential configuration to positively detect light/dark transitions. When a difference in raster intensity exists between the two phototransistors, the sawtooth raster appears at the output of the differential amplifier. A level shifter biases the sawtooth waveform to provide a CMOS-compatible input to the nonretriggerable monostable multivibrator. This monostable multivibrator triggers on the first pulse of the sawtooth and produces a stretched output pulse that lasts the duration of the stimulus, in order to prevent retriggering. The rising edge of the stretched pulse drives a second monostable multivibrator that produces a single, short duration, negative-true start pulse for the counter.

When complicated stimuli are used, it can take a significant amount of time for the graphic to "build" on the screen. To alleviate this effect, circuitry was devised (see Figure 4) that allows one of the TTL outputs on the timer board to switch the RGB signals to the secondary stimulus monitor on and off. In this manner, the screen can be blanked while the graphic builds and switched on when the graphic has been completed. In order to prevent picture "rolling" because of resynchronization of the monitor when the video is turned on, the horizontal and vertical synchronization signals remain applied to the monitor at all times, and only the RGB signals are switched. Methods of synchronizing the initiation of video to the start of the raster were investigated. Other researchers have used software synchronization methods (Creeger, Miller, \& Paredes, 1990) that work well in DOS but can result in errors when Windows is used. Synchronization for this system is accomplished through hardware that forces the RGB signal initiation to be coincident with the vertical sync signal presented to the monitor. The RGB switching circuitry consists of a Darlington transistor relay driver and three individual $50 \Omega$, coaxially shielded, high-frequency reed relays. These relays were chosen so that the video signals and, therefore, picture quality would not be af- 


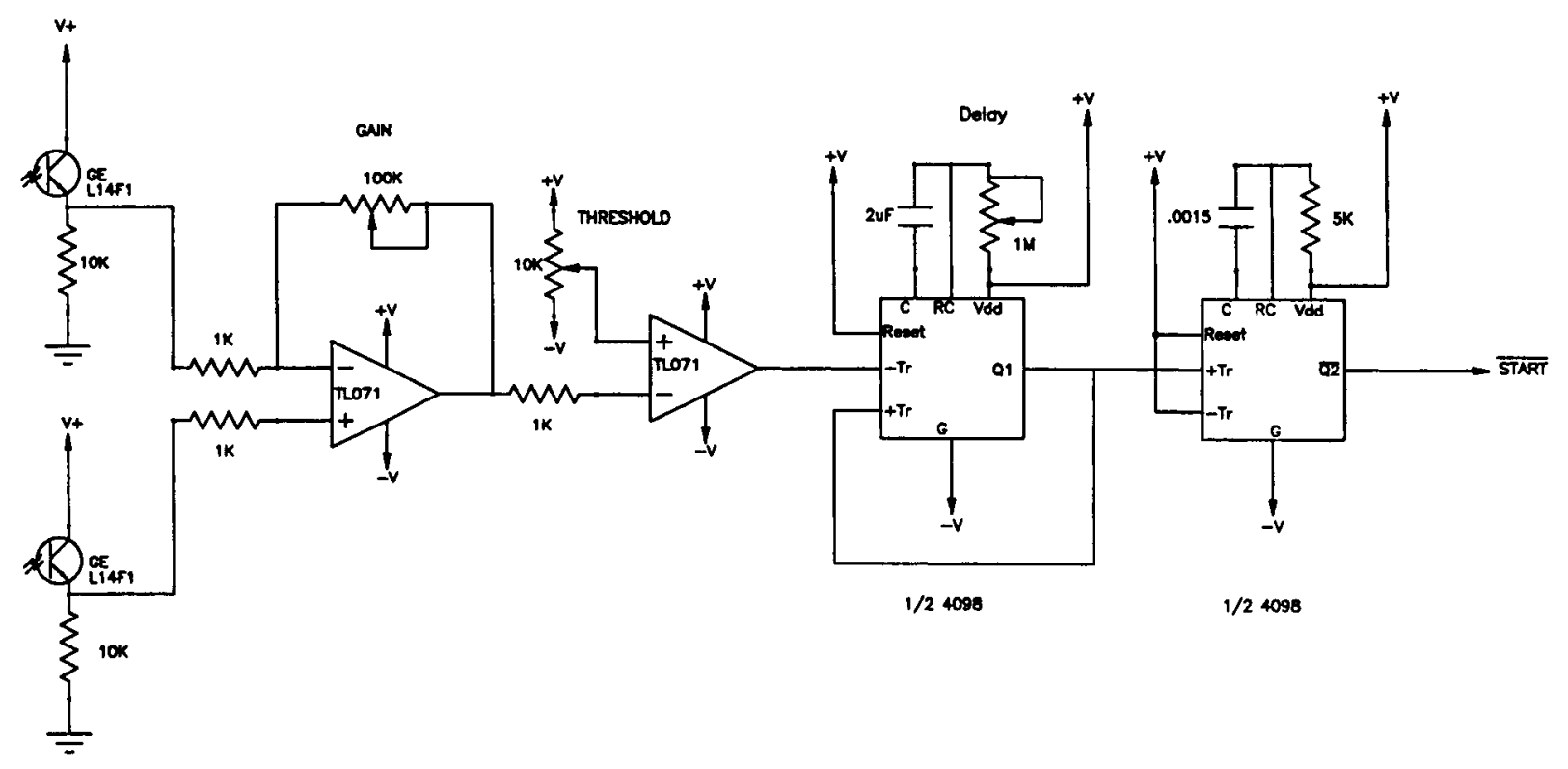

Figure 3. Photodetector circuitry.

fected. The synchronization circuitry consists of a D-latch that only allows the RGB signals to be turned on coincident with the rising edge of the vertical sync pulse. This circuitry is inserted in line with the stimulus monitor only, allowing experiment control graphics to be blanked from the stimulus monitor while still being displayed on the control monitor. As a convenient alternative to using the photodetector to sense stimulus onset, a fourth relay provides a start signal to the counter coincident with the application of RGB signals to the monitor. The timing resolution resulting from the use of the start signal from the photodetector circuitry and the video control circuitry is discussed later.

\section{Software}

All of the software was written to operate under Windows, because of the ability to transfer images between different applications with the clipboard and because of its familiarity to most PC users. Although this control software could have been written in many different languages, VisualBasic was chosen for several reasons: (1) familiarity and ease of program flow comprehension, (2) ability to easily handle images, (3) ability to access and display scalable fonts, and (4) ease of use by operators because of the ability to build user interfaces with familiar Windowsstyle icons and mouse control. The software driver for VisualBasic provided by the counter board manufacturer was used to provide commands for controlling the board function. One may choose, instead, to control the board operations through the use of a simple DLL written in C (Kemp, 1996; Titus, 1994), since it cannot be performed directly from VisualBasic (the Inp and Out commands were omitted). Figure 5 shows a flow chart of the backbone procedures for the software utilized here.
System operation proceeds as follows. The program initializes the three counters located on the counter/timer board first. This consists of setting the operational mode of each of the counters to mode 2 (rate generator mode). Jumpers on the board allow serial connection of all three counters and the clock. The first two counters are ungated and count continuously, whereas the third gated counter counts pulses only for the gate pulse duration. By loading counter preset values, counter 0 is set to divide by 100 , and counter 1 is set to divide by 10 , thus dividing the $10-\mathrm{MHz}$ clock down to $10 \mathrm{kHz}$. This sets the minimum time resolution to $100 \mu \mathrm{sec}$. Loading a preset value into counter 2 determines the total count time $\left(\mathrm{T}_{\text {MAX }}(\mathrm{sec})=100 \mu \mathrm{sec} \times\right.$ load value $)$. Valid presets are in the range of 1 to 65,535 ( 16 bits) and, when counter 2 is loaded with 65,535 , the maximum timing period becomes $6.5 \mathrm{sec}$. For example, if the clock was divided down to $1 \mathrm{kHz}$ instead, the resulting resolution would be $1 \mathrm{msec}$, with a maximum timing period of $65 \mathrm{sec}$. Therefore, improved counter accuracy and longer timing periods could be obtained through the use of a higher order counter, providing higher resolution and/or longer time periods.

Following the counter initialization, TTL I/O port D1 is initialized high (port $D 1$ provides a computer-generated stop signal to the counters through the circuitry of Figure 1). The timer functions provided within VisualBasic control the image display and image blanking times, because the precision of these times is less important. TTL I/O port D2 controls the video switching circuitry. Programs with printed character stimuli simply utilize the fonts provided in Windows to display words stored in arrays. Programs with complex image stimuli display named images that have been stored as "thumbnails." The display of the stimuli coincides with the starting of a soft- 


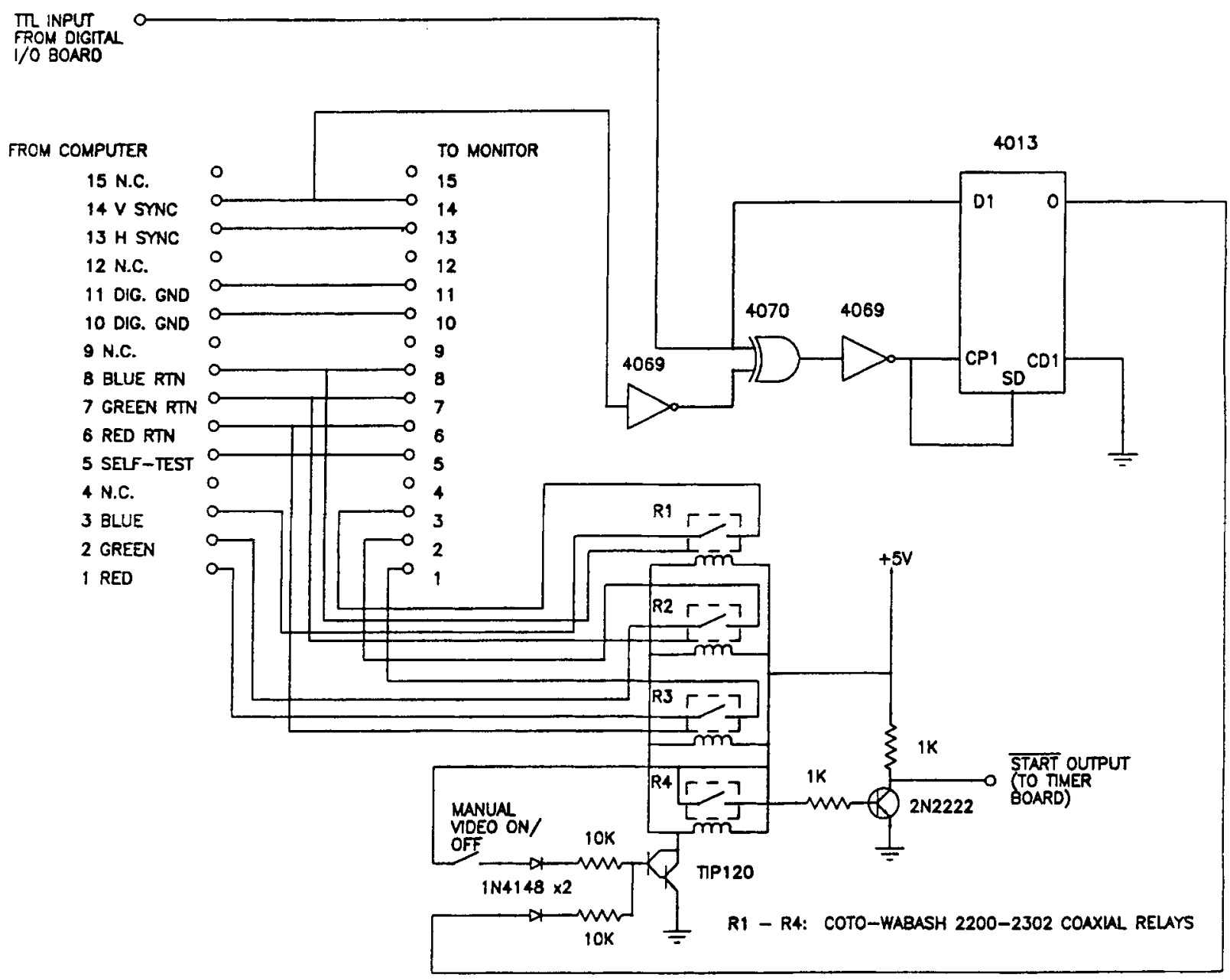

Figure 4. Screen blanking circuitry.

ware timer preset to the desired time interval between images. For most applications, random selection of the various stimulus patterns is utilized; however, previously randomized or ordered look-up tables may also be used. A second timer is preset with the image duration time and blanks the display for the remaining portion of the interval. When the image appears on the screen, a start pulse that initiates counter operation is provided by either the photodetector or the video switching. The counter stops either when the pushbutton is depressed or when port $\mathrm{Dl}$ is pulsed by the computer at the end of the time interval. The contents of counter 2 are then read, converted into a reaction time in milliseconds, and stored in an array. The counter is then reinitialized, and the process starts over. Once all of the images have been displayed, the response time data are tabulated, along with the time of day, the test sequence, and the name or type of image displayed. A summary is recorded for each test sequence by analyzing the data, to determine the number of correct and incorrect responses, along with the percentage accuracy. The final step in the program stores the data to the hard disk in ASCII format for later use and produces a backup file on floppy disk.

VisualBasic allows easy-to-use and professionallooking user interfaces to be constructed. The user interface is modified for each specific application but consists generally of the same major components for each application. For example, one particular application involves displaying eight randomly ordered complex fractal images with durations of $1 \mathrm{sec}$ on, $2 \mathrm{sec}$ off, with each test sequence lasting $5.5 \mathrm{~min}$. The user interface is not visible to the test subject, because the video to the secondary monitor is blanked whenever the control screen is displayed. The VisualBasic tools include graphical "buttons" and status indicators that can be placed on the screen, which interact with various aspects of the program. Buttons are provided that allow the researcher to start each test sequence, and status indicators show which tests have been run and how many times they have been repeated. Buttons are also provided to allow each of the images to be displayed manually for training purposes, to perform system tests, and to end the program. 


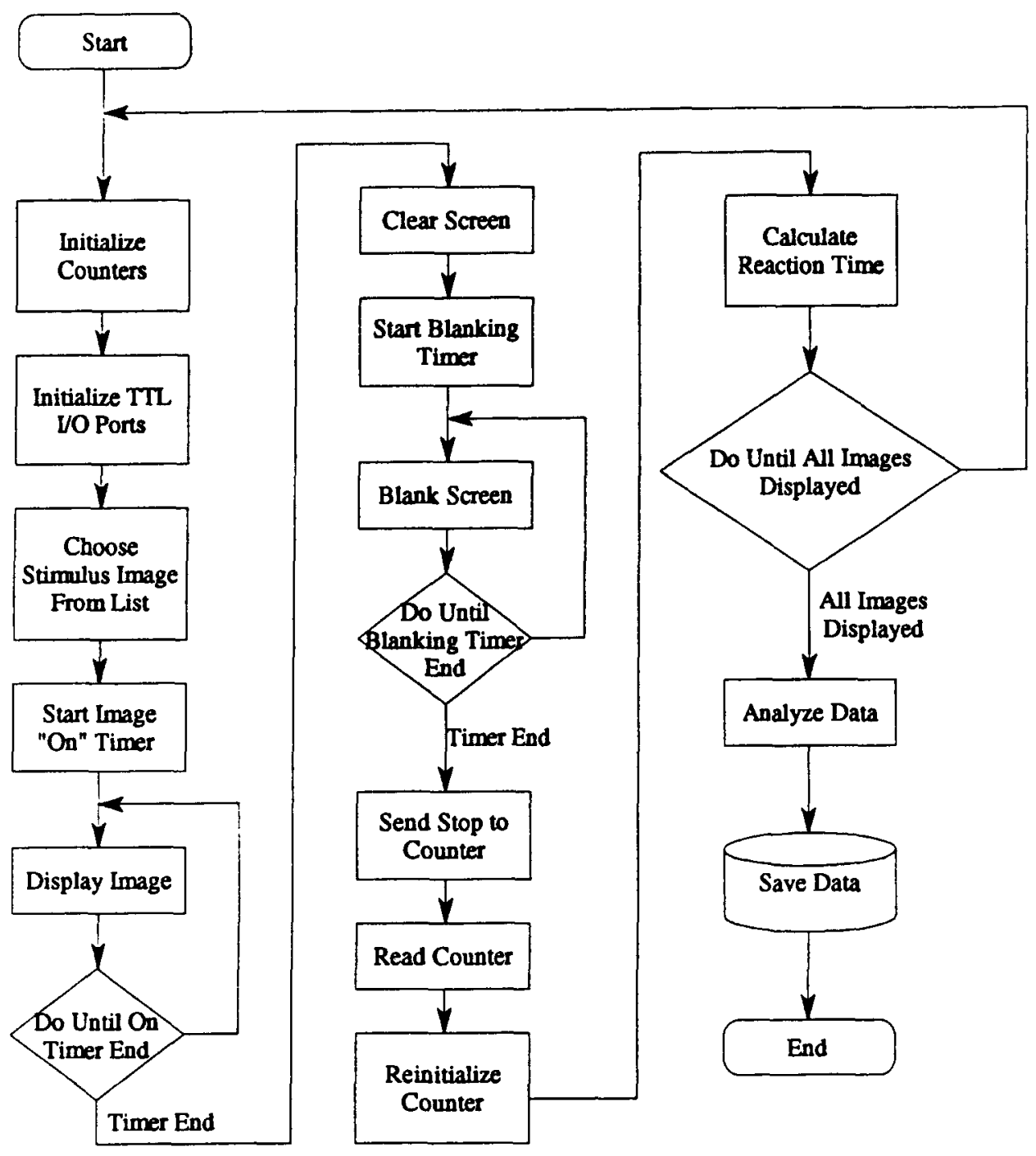

Figure 5. Flow chart.

\section{TIMING RESOLUTION}

As was indicated earlier, the presets loaded into the counters determine the maximum count time and the theoretical resolution of the hardware. The actual resolution of the counting system, therefore, is based on the accuracy of the time-base oscillator and the accuracy of the start and stop signals that control the counters. The manufacturer's $100-\mathrm{ppm}$ stability specification of the time-base oscillator used here results in a timing ambiguity of $\pm 0.1 \mu \mathrm{sec}$, which is several orders of magnitude below the $100-\mu \mathrm{sec}$ minimum resolvable time and, for all practical purposes, can be ignored.

The signal that is used to start the counting sequence also affects the timing resolution. Errors can result from time delays in the processing circuitry and in the detection of stimulus onset. There are different sources of er- rors for each of the two methods presented here for providing start signals to the counter.

Use of the photodetector start signal output results in errors owing to the response times associated with the detection of light and the processing circuitry. The response time of the photodetector circuitry was measured by using an LED driven by a pulse generator to illuminate one of the phototransistors while the other was darkened. The time difference between the signal applied to the LED and the start output signal was measured with an oscilloscope. The majority of the measured error results from the slow rise time of the phototransistors. Because of this, the response time was highly dependent on the comparator threshold and thus required threshold adjustment to minimize the delay times. Time delays of less than $100 \mu \mathrm{sec}$ were measured in this manner. In order to obtain an accurate start signal from the photodetector in 
actual use, the different characters or images must appear at the same location on the screen for each stimulus, so that the detector can be located in a fixed position to detect the intensity transition. If the image cannot be located in the same place on the monitor each time, a target block of several pixels must be lighted in order to give the detector a standard transition point to detect. Because of the raster scan frequency, there will be a fixed time difference between the lightings of various locations on the screen. Therefore, the photodetector gives a start pulse based on only one specific location on the screen, and time differences to other locations may need to be considered.

Use of the start signal output from the video switching circuitry results in different timing errors. The relays chosen have specified actuation times of $220 \mu \mathrm{sec}+100 /$ $-50 \mu \mathrm{sec}$. Therefore, the theoretical time difference between application of signal to the relays and application of video to the monitor will correspond to the specified relay actuation times. However, since the counter start signal is taken from the output of a fourth, identical relay, the theoretical difference between the start signal and the time that video is applied to the monitor is simply the specified variation of $150 \mu \mathrm{sec}$. The actual time response of the circuitry was measured by applying the output of a pulse generator to the TTL input of the video switching circuit. A voltage was applied to the contacts of each relay, and the time delay between the pulse generator signal and the relay outputs was measured with an oscilloscope. The four relays measured 174, 194, 172, and $170 \mu \mathrm{sec}$, indicating a maximum output differential of only $24 \mu \mathrm{sec}$. Therefore, timing resolution of $100 \mu \mathrm{sec}$ is achievable, utilizing either the photodetector or the video control circuitry start signal.

In actual use, the user of this system must still contend with other timing issues, depending on the application. For instance, one must be aware of the constraints that various raster scan rates have on the appearance of images. Finite, although fixed, delay times exist between the top left corner of the image and the bottom right corner of the image and can be of concern for certain applications (Paredes et al., 1990). The problem of producing very short duration stimuli exists with all raster scan displays because of finite refresh rates. Also, differences in phosphor persistence, brightness, and contrast levels between monitors can affect the visualization of various processes.

\section{CONCLUSIONS}

The combination of circuitry and software presented here results in a tachistoscopic measurement system that performs highly accurate timing measurements with a resolution of $100 \mu \mathrm{sec}$, using either of two methods for providing start signals. All of the original design goals were achieved: (1) Timing inaccuracies owing to varia- tions in computer hardware and software are avoided because of the use of external timing and synchronization hardware. Timing resolution of $100 \mu \mathrm{sec}$ was obtained, using either of two synchronization methods. (2) Timing measurements were made from VisualBasic running under the Windows operating system. (3) The selection of a timing board that connects to the parallel printer port allows this system to operate with any IBM-compatible $\mathrm{PC}$ running Windows, including laptops. (4) Microsoft VisualBasic was used to program all stimulation and timing functions on the computer, allowing simple user interfaces to be developed and simplifying program modifications. (5) Use of Windows and VisualBasic allows complex images to be imported through the clip board from other software packages via the cut-andpaste method.

In use, we have found that the measurement system operates smoothly. Especially because of the complexity of conducting a positron emission tomography (PET) experiment, the ease of use that the user interface provides was found to be invaluable. However, the methods described here have some limitations. Because this system was designed for specific PET applications, its flexibility for some users may be slightly limited. For other specific applications, the software may be the limiting factor and may require a person who can program in VisualBasic to modify the images, select different display timing, or change the user interface to reflect the particular study. Some technical ability in electronics is needed initially, because the system requires the assembly of some commercially unavailable hardware, although this is very simple. A laptop computer can be used as the console, but a second CRT video monitor is required for stimulus display, whether a laptop or a desktop system is used. In order to reduce the small amount of switching variability between coaxial relays, we are currently investigating highspeed, semiconductor RF switches as replacements in future versions.

The basic costs involved with this system are as follows. (1) The PPIO-CTR03 board is listed as $\$ 99.00$ from ComputerBoards, Inc., 125 High St., Mansfield, MA 02048, (508) 261-1123. (2) Microsoft VisualBasic Professional version 5.0 has an educational list price of $\$ 99.95$ and is available at almost any software house. (3) The MasterConsole VideoShare MCDRV 1 X2 video splitter has an educational list price of $\$ 251.75$ from Raritan Computer, Inc., 10-1 Ilene Court, Belle Mead, NJ 08502, (908) 874-4072. (4) The total parts cost for the other external circuitry is estimated to be less than $\$ 100.00$.

\section{REFERENCES}

Creeger, C. P., Miller, K. F., \& Paredes, D. R. (1990). Micromanaging time: Measuring and controlling timing errors in computercontrolled experiments. Behavior Research Methods, Instruments, \& Computers, 22, 34-79. 
DUtTA, A. (1995). Experimental RunTime System: Software for developing and running reaction time experiments on IBM-compatible PCs. Behavior Research Methods, Instruments, \& Computers, 27, 516-519.

GabrielsSON, J., \& JARVELla, R. J. (1990). How to obtain nearmillisecond precision on the IBM PC with visual stimuli not exceeding one printed line. Behavior Research Methods, Instruments, \& Computers, 22, 520-525.

Graves, R. E., \& Bradley, R. (1991). Millisecond timing on the IBM $\mathrm{PC} / \mathrm{XT} / \mathrm{AT}$ and PS/2: A review of the options and corrections for the Graves and Bradley algorithm. Behavior Research Methods, Instruments, \& Computers, 23, 377-379.

KemP, P. (1996, May 9). Program provides ISA-bus DLL for Windows. Electronic Design News, pp. 89-92.

Mogg, K., \& Bradley, B. P. (1995). Tachistoscopic applications of Micro Experimental Laboratory (MEL) used with IBM PC compatibles: Stimulus and response timing issues. Behavior Research Methods, Instruments, \& Computers, 27, 512-515.

Palya, W. L., Walter, D. E., \& Chu, J. Y. M. (1995). An inexpensive 1-millisecond experiment control interface for IBM PCs and its userfriendly control language. Behavior Research Methods, Instruments, \& Computers, 27, 129-130.

Paredes, D. R., Miller, K. F., \& Creeger, C. (1990). Graphic precision: Controlling stimulus displays on IBM PC-compatible computers. Behavior Research Methods, Instruments, \& Computers, 22, 319-322.

Rodgers, K. A., Schneider, W., Pitcher, E., \& Zuccolotto, A. (1995). MEL Professional: Language Reference Guide (Vol. 1). Pittsburgh, PA: Psychology Software Tools, Inc.

Segalowitz, S. J., \& Graves, R. E. (1990). Suitability of the IBM XT, $\mathrm{AT}$, and PS/2 keyboard, mouse, and game port as response devices in reaction time paradigms. Behavior Research Methods, Instruments, \& Computers, 22, 283-289.

Titus, J. (1994, February 17). VisualBasic does I/O. Electronic Design News, p. 56.

(Manuscript received August 22, 1997; revision accepted for publication February 9, 1998.) 